\title{
Comprehensive Analysis of a 14 Immune-related Gene Pairs Signature to Predict the Prognosis of Gastric Cancer and Immune Features
}

\section{Chuan Liu}

Department of Medical Oncology, the First Hospital of China Medical University, Shenyang, 110001, China

\section{Bo Chen}

The First Clinical College, Wenzhou Medical University, Wenzhou, 325035, China

\section{Zhangheng Huang}

Department of Orthopaedic Surgery, Affiliated Hospital of Chengde Medical University, Chengde, 067000, China

\section{Chuan $\mathrm{Hu}$}

Department of Joint Surgery, the Affiliated Hospital of Qingdao University, Qingdao, 266071, China

\section{Liqing Jiang}

Department of Medical Oncology, the First Hospital of China Medical University, Shenyang, 110001, China

Chengliang Zhao ( $\sim 38221965 @ q q . c o m$ )

Chengde Medical University

\section{Research}

Keywords: Immune-related gene pairs, Gastric cancer, Immune cells, Prognosis, Nomogram

Posted Date: August 19th, 2020

DOI: https://doi.org/10.21203/rs.3.rs-60067/v1

License: @ (i) This work is licensed under a Creative Commons Attribution 4.0 International License. Read Full License

Version of Record: A version of this preprint was published at International Immunopharmacology on December 1st, 2020. See the published version at https://doi.org/10.1016/j.intimp.2020.107074. 


\section{Abstract}

Background: As a new method for predicting tumor prognosis, the predictive effect of immune-related gene pairs (IRGPs) has been confirmed in several cancers, but there is no comprehensive analysis of the clinical significance of IRGPs in gastric cancer (GC).

Methods: The clinical and gene expression profile data GC patients were obtained from the GEO database. Based on the ImmPort database, differently expressed immune-related genes (DEIRGs) events were determined by a comparison of GC samples and adjacent normal samples. Cox proportional regression was used to construct an IRGPs signature, and its availability was validated with three external validation sets. In addition, we explored the association between clinical data and immune features and established a nomogram to predict outcomes of GC patients.

Result: A total of 88 DEIRGs were found in GC from the training set, which formed 3828 IRGPs. 14 overall survival (OS)related IRGPs were used to construct the prognostic signature. As a result, the patients in the high- risk group have a poorer OS compared with the low-risk group. In addition, the fraction of CD8+ T cells, plasma cells, T cells CD4 memory activated, and macrophages M1 were higher in the high-risk group. The expression of two immune checkpoints, CD276 and VTCN1, was significantly higher in the high-risk group. Based on the independent prognostic factors, a nomogram was established and showed excellent performance.

Conclusion: The 14 OS-related IRGPs signature was associated with the OS, immune cells, and immune checkpoints of GC patients, which can provide the basis of related immunotherapy.

\section{Background}

From the worldwide epidemiological survey, the incidence and mortality of gastric cancer (GC) rank fifth and third among all cancers, respectively [1, 2]. In recent years, a series of treatments of GC has been continuously optimized, including microsurgery, immunotherapy, and molecular targeted therapy, and the prognosis of patients has been effectively improved [3-5]. However, the five-year survival rate is still lower than $25 \%$, and one of the reasons is that most patients are already in the advanced stage or metastatic status when first diagnosed [6, 7]. Additionally, since patients with the same stage sometimes have a different prognosis, it is essential to understand the mechanism of GC and find relative prognostic predictors to estimate disease prognosis and guide the therapy. It was reported in previous studies that several clinicopathologic factors, genes, microsatellite instability, and noninvasive markers can be used as prognostic factors, but most of them lack clinical practice or have limitations [8-10]. Therefore, effective predictors that can be used for accurate prediction and prognosis improvement of GC patients are highly needed.

Recent studies have shown that immune-related genes (IRGs) are involved in the regulation of the immune system, which plays an important role in the generation and progression of cancers [11, 12]. Increasing pieces of evidence have reported important characteristics of IRGs in the complex regulatory network of cancers, which can be used as biomarkers to predict the prognosis of cancer patients [13]. For instance, Qiu et al. [14] and Yang et al. [15] have found that IRGs were related to the prognosis of GC patients and established an effective prediction model to predict their prognosis. However, with the upgraded data of gene sequencing methods, new sequencing and traditional sequencing require appropriate standardization of gene expression levels, which is a challenge for IRGs-based models currently. Luckily, researchers have proposed new methods to eliminate data processing limitations, such as standardization and scaling based on the relative ranking of gene expression levels, which has produced reliable results in various studies [16-18].

Therefore, in this study, we integrated one of the new methods-immune-related gene pairs (IRGPs) as predictors to comprehensively study their effects on prognosis, immunity, and immune checkpoints of GC patients. 


\section{Methods}

\section{Data acquisition and processing}

Retrospective research and comprehensive analysis of the relationship between IRGPs and the prognosis of GC patients were performed based on public data sets. The clinical data of GC patients and the corresponding gene expression profile data were obtained from the Gene Expression Omnibus (GEO) database (http://www.ncbi.nlm.nih.gov/geo). In total, six GC cohorts were found, including GSE13911, GSE118916, GSE84437, GSE13861, GSE26901, and GSE66229. Specifically, GSE13911 and GSE118916 were used to identify differently expressed immune-related genes (DEIRGs) between GC and normal tissues. GSE84437 set was used to construct a prognostic signature and the remaining three sets were used as validation sets to verify the effectiveness of the IRGPs signature. For training and validation sets, patients without complete survival data will not be included in this study.

\section{Identification of tumor-associated IRGs and enrichment analysis}

There are gene expression profiles of both GC tissues and adjacent normal gastric tissues in GSE13911 (T:38, N:31) and GSE118916 (T:15, N15). In previous studies, comparing biomarkers in different pathological conditions to determine tumor-related biomarkers has been widely used [19]. Thus, we performed the analysis by the GEO2R tool, and those genes with adjusted $\mathrm{P}<0.05$ and $|\mathrm{Log} 2 \mathrm{FC}|>1$ were defined as differentially expressed genes (DEGs). Then, we downloaded IRGs from the ImmPort database (https://www.immport.org/shared/)[20] to identify DEIRGs. After that, the interaction DEIRGs between GSE13911 and GSE118916 were used for later studies.

To initially understand the mechanism and function involved in DEIRGs, these DEIRGs were then incorporated into the enrichment analysis, including Gene Ontology (GO) and Kyoto Encyclopedia of Genes and Genomes (KEGG) pathways with metascape [21].

\section{Construction of a prognostic signature based on IRGPS}

It was reported that IRGPs have a robust prognostic ability in different cancers [17, 22]. the cohort of GSE84437 ( $n=433)$ that contain both IRGs and clinical data was used as the training set to form various IRGPs (Supplementary file1). And each IRGP was formed by comparing the gene expression level of a specific sample or profile of IRGs. Specifically, in a pairwise comparison, if the expression of the first element is greater than the latter element, the output is 1 . Otherwise, the output is 0 . This method of forming IRGPs can avoid the difficulties caused by different gene expression profiles, and does not need to be standardized for personal evaluation. And those IRGPs with a constant value (more than $80 \%$ of the samples are assigned the same score on the training set) would be eliminated in the subsequent model construction because they do not provide enough discriminatory information [23].

To identify the prognostic value of IRGPs in GC patients, the univariate Cox analysis was used to identify OS-related IRGPs in the training set, and those with $\mathrm{p}<0.05$ were considered as candidate prognostic IRGPs. Then, based on the candidate IRGPs identified in the univariate Cox analysis, LASSO penalized Cox regression was performed to choose the most significant pairs [24]. Finally, multivariate Cox analysis was used to select the most appropriate OS-related pairs and construct an IRGPs signature. To assess the performance of the IRGPs signature for GC patients, we generated receiver operating characteristic (ROC) curves at 1-, 3-, 5-years [25]. In addition, based on the individual risk score, GC patients were divided into high- and low-risk groups by the median risk score. Then, the Kaplan-Meier (K-M) survival curve was used to compare the differences in OS between the two groups with the log-rank test. Moreover, the subgroup analysis was applied for validation of the signature in different subgroups, including age (age $₫ 65$, age $₫ 65)$, gender (female, male), $\mathrm{N}$ stage (N0-1, N3-4), T stage (T1-3, T4).

\section{Validation of the expression of IRGPs in Oncomine}


Oncomine is a cancer microarray database and integrated data-mining platform (http://www.oncomine.org) that contains more than 65 gene expression data sets comprised of 48 million gene expression measurements. By using expression data and clinical data from this database, all the IRGs of OS-related IRGs in the prognostic signature were explored to verify their differential expression in GC and normal gastric tissues.

\section{Validation of the IRGPs signature}

In order to verify the prognostic value of the IRGPs signature, three validation sets were applied, including GSE13861 $(n=65)$, GSE26901 $(n=109)$, and GSE66229 $(n=300)$. According to the risk score formula in the training cohort, the risk score of each patient in the validation cohort was calculated. Based on the optimal cut off value identified in the training cohort, all patients in the validation cohort were divided into high- and low-risk groups. The K-M survival curves with the log-rank test were used to compare the differences of prognosis between these two groups.

\section{The explosion of immune features of IRGPs in GC}

CIBERSORT is a common algorithm to obtain cell composition from volume tumors or gene expression profiles [26], which was used in our study to understanding the enrichment of immune cells in different risk groups. For each sample, CIBERSORT infers the relative proportion of 22 infiltrating immune cells, including T cells, B cells, natural killer cells, macrophages, plasma cells, neutrophils, dendritic cells (DCs) and so on. Additionally, associations between IRGPs and a variety of immune checkpoints were explored. The associations between risk groups and infiltration levels of 22 kinds of immune cells and immune checkpoints were assessed using the Wilconson's rank-sum test or Kruskal-Wallis test. A Circos diagram was generated by R software to display the genes in IRGPs on the corresponding chromosomes and visualize their interactions.

\section{Development of a nomogram based on the immune and clinical prognostic factors}

In order to improve the clinical utilization of IRGPs signature for GC patients, we conducted the univariate Cox analysis based on risk score and clinical variables in the training set, including age, sex, T stage, and $\mathrm{N}$ stage. As for the $\mathrm{N}$ stage, it was found that the prognosis of GC patients with NO stage was significantly better than other stage patients in previous studies, and the prognosis of N1, N2, and N3 differed less [27]. Therefore, we divided the N stage into N0 and N1-3 groups. Then, the variables with $\mathrm{p}<0.05$ were included in the multivariate Cox analysis to identify the independent OSrelated factors. Based on independent OS-related factors, a nomogram was developed by rms package. The concordance index (C-index), the calibration curve, and decision curve analyses (DCA) were used to evaluate the performance of the nomogram.

\section{Statistical analysis}

All statistical analyses and graphics in our study were performed using R software(version 3.6.1). The student's t-test or Wilcoxon rank-sum test was used to comparing the mean standard error of the mean of continuous variables among groups. The Cox proportional hazards regression model was used for univariate and multivariate survival analysis. The gene model was conducted with multivariate Cox analysis, and the ROC curves were conducted by "survival ROC". A pvalue of less than 0.05 (two sides) was considered as statistical significance.

\section{Results}

\section{Identification of DEIRGs and relative enrichment analysis}

To better find IRGs specifically related to GC, we analyzed the difference in gene expression between normal tissues and tumor tissues. Totally, there are 303 DEIRGs in the GSE13911 set, including136 upregulated and 167 downregulated 
(Supplementary file2). Meanwhile, 147 DEIRGs were confirmed in the GSE118916 set, including 91 upregulated and 56 downregulated (Supplementary file3). After the intersection of these two DEIRGs sets, a total of 88 IRGs were confirmed, which included 47 upregulated and 41 downregulated IRGs (Supplementary file4 and Supplementary Fig. 1). Interestingly, among the 88 interaction IRGs, some were also differentially expressed in colorectal cancer and ovarian cancer as previously reported $[18,28]$. For example, the IRGs of MMP12, PLAU, ILIR2, and NR3C2 were associated with GC and ovarian cancer, while CCL8, MMP9, GAL, PLAU, BIRC5, and LIF were related to both GC and colorectal cancer. The comparison indicated the role of these IRGs in tumorigenesis, which should be paid more attention in later research and experiments.

The results of GO analysis were illustrated in Fig. 1A, which showed that specific GO categories were significantly related to DEIRGs, like leukocyte migration, receptor ligand activity, and positive regulation of MAPK cascade. In addition, some KEGG pathways related to GC development were enriched (Fig. 1B), including neutrophil mediated immunity, neutrophil activation, and regulation of inflammatory response. In a word, the enrichment analysis suggested DEIRGs play an immune role in GC, which helped reveal the potential modification mechanisms of protein function.

\section{Development and validation of the prognostic IRGPs signature}

The gene expression profiles of 88 DEIRGs were obtained from the set of GSE84437, which was used to establish an IRGPs signature and the IRGPs-clinicopathologic nomogram. 1546 IRGPs were retained and selected as the initial candidate IRGPs after eliminated those with a constant value (Supplementary file5). With the univariate Cox analysis, 234 OS-related IRGPs were identified (Supplementary file6), and the 20 most significant IRGPs related to the prognosis of GC are shown in Supplementary Fig. 2A, including 12 negative and 8 positive pairs. Then, we performed Lasso Cox proportional hazards regression on the training set and selected 27 IRGPs (Supplementary file7 and Supplementary Fig. 2B-2C), which were included in the multivariate Cox analysis. Finally, 14 IRGPs were selected to construct a prognostic signature (Table 1). As shown in the coefficients in the table, three IRGPs were positively correlated with patient prognosis, while eleven were negatively correlated.

Table 1 The information of IRGPs signature 


\begin{tabular}{|c|c|c|c|c|c|}
\hline & Immune processes & IRG 2 & Immune processes & Coe & $\begin{array}{l}P \\
\text { value }\end{array}$ \\
\hline $\mathrm{CHGA}$ & Cytokines & INBHA & Cytokines & 0.374 & 0.065 \\
\hline CHGB & Cytokines & OSMR & Cytokine Receptors & -0.541 & 0.002 \\
\hline CXCL14 & Antimicrobials & CXCL6 & Antimicrobials & -0.605 & 0.000 \\
\hline CXCL9 & Antimicrobials & GREM1 & Cytokines & -0.401 & 0.030 \\
\hline F2RL1 & Antimicrobials & TNFRSF12A & Cytokine Receptors & -0.394 & 0.010 \\
\hline FAM3B & Cytokines & GREM1 & Cytokines & -0.572 & 0.007 \\
\hline FAM3B & Cytokines & TLR3 & Antimicrobials & 0.642 & 0.005 \\
\hline FCER1G & NaturalKiller_Cell_Cytotoxicity & THBS1 & Antigen_Processing_and_Presentation & -0.709 & 0.003 \\
\hline GAL & Cytokines & PLA2G2A & Antimicrobials & 0.432 & 0.006 \\
\hline INHBA & Cytokines & NRG4 & Cytokines & -0.449 & 0.006 \\
\hline MMP12 & Antimicrobials & OLR1 & Antimicrobials & -0.440 & 0.005 \\
\hline MMP9 & Antimicrobials & TNFRSF12A & Cytokine Receptors & -0.280 & 0.057 \\
\hline OASL & Antimicrobials & PGC & Antimicrobials & -0.541 & 0.006 \\
\hline REG1A & Cytokines & SPP1 & Cytokines & -0.510 & 0.003 \\
\hline
\end{tabular}

Then, based on the prognostic model, the risk score of each GC patients was calculated, and all patients were stratified into high- and low- risk groups according to the median of the risk score. As a result, the patients in the high-risk group have an extremely poorer OS compared with the low-risk group (P囚0.0001) (Fig. 2A), and the specific risk score and survival status of each patient were shown in Fig. 2C-2D. The corresponding time-dependent area under the curves (AUCs) of ROC of 1-, 2-, and 3-years for OS were $0.781,0.767$, and 0.776 , respectively, which means that the IRGPS signature can serve as a useful prognostic predictor for GC patients (Fig. 2B).

\section{Validation of the expression of IRGs in Oncomine}

In this study, 14 IRGPs were incorporated into the final OS signature, including 24 IRGs. The expressions of these IRGs in GC and normal gastric tissues were downloaded from the Oncomine database. As the result, there were 21 IRGs showed significantly different expression levels between GC and normal tissues (except for FAM3B, TNFRSF12A, and CHGA), which indicated the important role of these IRGs in the development of GC and guaranteed the accuracy of results (Supplementary Fig. 4).

\section{External validation of the IRGPs signature}

To study whether the IRGPs signature has similar prognostic value in different data sets, we applied the risk score at three different cohorts (GSE13861, GSE26901, and GSE66229) as external validation sets. The patients in each validation set were divided into high- and low-risk groups by the optimal cut off identified in the training set, and the survival time was compared. Similar to the results of the training set, patients in the high-risk group have a shorter OS than those in the lowrisk group (P®0.05) (Fig. 3). More interestingly, we also find that patients in the high-risk group have worse relapse-free survival (RFS) than low-risk patients.

\section{Potential mechanisms of IRGPs in GC}


It was obvious that IRGPs was closely associated with the prognosis of GC patients, to further explore the potential mechanisms, we analyzed the expression of immune checkpoints and the distribution of immune cells in the two risk groups. As shown in Fig. 4B, the expression of five immune checkpoints were significantly different between the two groups. Among them, the expression of CD276 (B7-H3) and VTCN1 in the high-risk group was significantly higher than that in the low-risk group, while the expression of PD-1, PD-L1, and CTLA-4 was reversed. This result indicated that the high expression of CD276 and VTCN1 may promote the expression of IRGs, leading to a poor prognosis, which is consistent with previous studies $[29,30]$. At the same time, it was shown that the fraction of CD8+ T cells, plasma cells, $T$ cells CD4 memory activated, and macrophages M1 were significantly higher in the high-risk group than low-risk group (Fig. 4A). Among the 14 IRGPs, the risk score of 1 in GALIPLA2G2A, CHGA|INHBA, and FAM3B|TLR3 was associated with high risk in GC, which suggested their potentially harmful role in GC patients' prognosis. And the remaining were opposite (Fig. 4C). At the same time, the N2-3 stage and T3-4 stage were enriched in the high- risk group, but there were no differences in sex and age between these two groups.

To understand the detailed relationship of risk score with immune microenvironment and clinical features. We found that a high level of CHGA|INHBA, CXCL9|GREM1, CXCL14|CXCL6, and INHBA|NRG4 was associated with a high level of B cells memory and low level of T cells CD4 memory activated (Supplementary Fig. 5A-5D). Then, it was shown that the risk score was higher in T3-4 and N1-2, which suggested the negative influence of risk score in the progress of GC (Supplementary Fig. 5E-5F).

To further explore the interaction between the related IRGs, a Circos diagram was developed, and the location of each IRG is shown in Fig. 4D. Although there were complex and diverse interrelationships between these genes, no link was found in each IRGP. Hence, it was more accurate to analyze the role of IRGPs in prognosis.

\section{Construction of an IRGPs-clinical nomogram}

Several clinical variables were found to be correlated with GC patients' prognosis like tumor site, tumor size, and TNM stage [31, 32]. Therefore, to predict the prognosis of GC patients more accurately, we used clinical variables and the risk score of IRGPs signature to establish a nomogram in the training set. The results indicated that age, $\mathrm{T}$ stage, $\mathrm{N}$ stage, and risk score were independently associated with OS (Fig. 5A). Then, a prognostic nomogram was developed based on these independent factors (Fig. 5B). The C-index for the OS nomogram was 0.719 (95\% Cl: 0.686 to 0.752 ), and the DCA and calibration curves of this nomogram for 1-, 2-, and 3-years were also established (Fig. 5C-F), which showed high credibility.

\section{Discussion}

GC is a highly malignant tumor, and its aggressiveness and high recurrence rate make the prognosis of GC patients still a challenge. Sensitive and reliable prognostic biomarkers are needed to identify those patients who have a poor prognosis and can benefit from the treatment. Although many gene-based prognostic signatures have been found to predict the prognosis of patients [33-35], their infeasibility and difficulty in measurement have led to lower clinical applicability. In the present study, a prognostic signature based on 14 IRGPs was developed and successfully validated in three independent datasets. Further research suggested that the differences between immune cells and immune checkpoints in different risk groups so that we supposed them to have predictive effects and to be used as new therapeutic targets. Finally, an IRGPsclinical nomogram was established and showed an accurate predictive ability of the OS for GC patients.

Last year, Jiang et al [36] comprehensively studied the relationship between the prognosis of GC and IRGs and their corresponding immunological characteristics. Considering the technical deviations caused by different sequencing platforms, the previous prognostic model requires the standardization of expression profiles, which makes data analysis more complex. However, the prognostic signature in our study was based on gene pairs, not just on genes. This is a new 
method of data analysis, which does not need to consider the technical deviation of different platforms [37], nor does it require scaling and normalization, but by pairwise comparison of gene expression values. This method was revealed to be reliable in colon cancer [38]. In the current study, IRGPs not only were used as a whole to predict the prognosis of GC patients but can simplify the detection procedure to some extent. For example, both CXCL9 and GREM1 are upregulated in GC. The increased level of CXCL9 is correlated with advanced disease stage and the recurrence of GC patients [39, 40], and GREM1 expression has been experimentally confirmed to be significantly associated with tumor growth and lymphatic metastasis of GC, leading poor survival [41]. These two genes form an IRGP in our study, and when the expression level of the former is lower than that of the latter, a high-risk score can be obtained resulting in a poor prognosis. The result only needs a simple test to compare the expression level, no specific expression data, and its feasibility is better than the traditional detection of IRGs.

IRGs were proved to have a positive correlation with some immune cells, which can affect the prognosis of patients [42, 43]. Thus, we studied the difference of immune cells distribution in high- and low-risk groups of the IRGPs signature. From the results, the infiltration level of plasma cells, T cells CD8, T cells CD memory activated, and macrophages M1 was significantly higher in the low-risk group, indicating that the immune response in tumor patients plays a protective role. CD4 + T cells can recognize tumor antigens and activate CD8 + T cells, thereby producing cytotoxic effects on tumor cells [44]. In previous studies, it was also found that the activation of $T$ cells is positively correlated with the prognosis of GC patients $[45,46]$. Plasma cells are a class of cells that can produce antibodies, and their number is significantly less in gastric cancer tissues compared to non-cancerous gastric mucosa, which suggested their inhibition role in tumor development [47]. Also consistent with our results, high infiltration of M1 macrophages in the tumor is associated with an increase in the patient's overall survival rate, while the effect of M2 macrophages is reversed [48, 49].

n our research, we also explored the immune checkpoints' role in risk groups. It was obvious that the expression of PD-1, PD-L1, and CTLA-4 was significantly higher in the low-risk group, which means that their high expression may improve patient prognosis. Immune checkpoints are the main targets of immunotherapy, which not only affect the tumor immune cell infiltration but also are related to the prognosis of patients. Previous studies reported that high levels of PD-L1 and CTLA-4 prolonged survival time of GC patients, which was associated with the increased infiltrating density of immune cells $[50,51]$. However, good results for patients with increased expression of these immunosuppressive molecules are counterintuitive and contrary to the results reported by many malignancies $[52,53]$. Therefore, we hypothesized that the upregulation of these immunomodulatory molecules may reflect an effect of inducing immune activation of immune cells. When the immune system is activated, it will still attack the tumor and show a survival advantage and nonactivated immunity.

The nomogram is a practical and intuitive evaluation tool, which has been widely used in previous studies [54-56]. Clinical factors we included have been used as risk factors in other studies [57-59], indicating that the prognostic value of these characteristics has been widely recognized. After univariate and multivariate Cox regression analysis, we found that the risk score of IRGPs was also an independent risk factor for the prognosis of GC. Therefore, incorporating it into the establishment of the nomogram can more accurately predict the prognosis of patients, and DCA and calibration curves showed the effectiveness of this nomogram. Although there have been previous nomograms combining clinical variables and genomes like mRNAs and specific genes to predict the prognosis of GC $[60,61]$, this is the first study to combine IRGPs signature to predict the prognosis of GC patients, which provides new ideas for the nomogram study.

There are still several limitations to our study. On the one hand, although we applied different external data sets for verification, it was still a retrospective study that lacks long follow-up and corresponding clinical trials. On the other hand, guidelines of ASCO recommended evaluating all clinical-pathological risk factors as much as possible in the prognostic model to improve the risk prediction of patients [62]. Unfortunately, due to the limitations of public databases, only a fraction of clinical variables were available, and other variables that may affect the prognosis of patients were not 
included, such as $\mathrm{M}$ stage and treatment status. Therefore, it is necessary to obtain more factors to construct a more accurate nomogram.

\section{Conclusion}

In summary, we comprehensively analyzed the prognostic role of the IRGPs signature, which could provide an effective method to assess the risk of GC patients. Besides, to understand the potential mechanisms and find the related targets of immunotherapy, the value of immune cells and immune checkpoints in different risk groups based on the IRGPS signature was explored. Finally, combined with the independent clinical factors, a nomogram was developed to accurately predict the prognosis, which will help in more effective treatment and management.

\section{Abbreviations}

IRGPS: immune-related gene pairs; GC: gastric cancer; DEIRGs: differently expressed immune-related genes; OS: overall survival; IRGs: immune-related genes; GEO: Gene Expression Omnibus; DEGs: differentially expressed genes; GO: Gene Ontology; KEGG: Kyoto Encyclopedia of Genes and Genomes; ROC: receiver operating characteristic; K-M: Kaplan-Meier; DCs: dendritic cells; C-index: concordance index; DCA: decision curve analyses; AUCs: area under the curves; RFS: relapsefree survival;

\section{Declarations}

\section{Ethics approval and consent to participate}

The research didn't involve animal experiments and human specimens, no ethics related issues.

\section{Consent for publication}

Not applicable.

\section{Availability of data and materials}

The data of this study are from GEO and ImmPort database.

\section{Competing interests}

The authors declare that they have no competing interests.

\section{Funding}

We received no external funding for this study.

\section{Authors' Contributions}

$\mathrm{C} \mathrm{L}, \mathrm{B} \mathrm{C}$, and $\mathrm{CL} \mathrm{Z}$ conceived of and designed the study. C L, ZH H and C H performed literature search. ZH H and C H analyzed the data. B C ZH H and LQ J generated the figures and table. C L wrote the manuscript and CL Z critically reviewed the manuscript. $\mathrm{CL} \mathrm{Z}$ and $\mathrm{C} \mathrm{H}$ supervised the research. All authors have read and approved the manuscript.

\section{Acknowledgements}

None 


\section{References}

1. F B, J F, I S, RL S, LA T, A J: Global cancer statistics 2018: GLOBOCAN estimates of incidence and mortality worldwide for 36 cancers in 185 countries.CA: a cancer journal for clinicians 2018, 68:394-424.

2. J F, M C, I S, C M, DM P, M P, A Z, F B: Estimating the global cancer incidence and mortality in 2018: GLOBOCAN sources and methods.International journal of cancer 2019, 144:1941-1953.

3. J W, C L, X Z, J Z: Adjuvant therapy in resectable gastric cancer-the CRITICS trial.The Lancet Oncology 2018, 19:e330.

4. YJ B, E VC, A F, HC C, L S, A S, F L, A O, Y O, T S, et al: Trastuzumab in combination with chemotherapy versus chemotherapy alone for treatment of HER2-positive advanced gastric or gastro-oesophageal junction cancer (ToGA): a phase 3, open-label, randomised controlled trial.Lancet (London, England) 2010, 376:687-697.

5. CS F, K S, M DB, S L, SE A-B, E VC, DH I, M A, I C, J L, et al: Ramucirumab with cisplatin and fluoropyrimidine as firstline therapy in patients with metastatic gastric or junctional adenocarcinoma (RAINFALL): a double-blind, randomised, placebo-controlled, phase 3 trial. The Lancet Oncology 2019, 20:420-435.

6. P C: Gastric cancer: overview.Gastroenterology clinics of North America 2013, 42:211-217.

7. L S, YS S, HM H, TJ P, B S, KH Y, YH Y, T S, HK Y, X Z, et al: Management of gastric cancer in Asia: resource-stratified guidelines. The Lancet Oncology 2013, 14:e535-547.

8. L W, XZ C, K Y, ZX C, B Z, JP C, ZG Z, XM M, JK H: Prognostic value of cancer stem cell marker CD133 expression in gastric cancer: a systematic review.PloS one 2013, 8:e59154.

9. MH M, EM E-O: Genetics of gastric cancer.Nature reviews Gastroenterology \& hepatology 2014, 11:664-674.

10. W W, XL C, SY Z, YH X, WH Z, K L, XZ C, K Y, B Z, ZX C, et al: Prognostic significance of preoperative serum CA125, CA19-9 and CEA in gastric carcinoma.Oncotarget 2016, 7:35423-35436.

11. AJ G, AM N, CL L, SV B, W F, D K, VS N, Y X, A K, CD H, et al: The prognostic landscape of genes and infiltrating immune cells across human cancers. Nature medicine 2015, 21:938-945.

12. L L, Y O, W W, D H, Y Z: The landscape and prognostic value of tumor-infiltrating immune cells in gastric cancer.PeerJ 2019, 7:e7993.

13. WH F, L Z, C S-F, G K: The immune contexture in cancer prognosis and treatment.Nature reviews Clinical oncology 2017, 14:717-734.

14. H Q, X H, C H, B Y, Y L, J L: Identification and Validation of an Individualized Prognostic Signature of Bladder Cancer Based on Seven Immune Related Genes.Frontiers in genetics 2020, 11:12.

15. W Y, Z L, Y L, J M, M Y, J X, J X: Immune signature profiling identified prognostic factors for gastric cancer.Chinese journal of cancer research = Chung-kuo yen cheng yen chiu 2019, 31:463-470.

16. M H, M N, R K, A W-B, L S, JX Z, R K, SA K, S H, I S: Gene-pair expression signatures reveal lineage control.Nature methods 2013, 10:577-583.

17. B L, Y C, M D, R L: Development and Validation of an Individualized Immune Prognostic Signature in Early-Stage Nonsquamous Non-Small Cell Lung Cancer.JAMA oncology 2017, 3:1529-1537.

18. J W, Y Z, J Z, Q W, W W: Development and validation of an immune-related gene pairs signature in colorectal cancer.Oncoimmunology 2019, 8:1596715.

19. ZX L, ZQ Z, ZH W, LL Z, F L, L L, RQ L, XD H, JW L, FP C, et al: Comprehensive characterization of the alternative splicing landscape in head and neck squamous cell carcinoma reveals novel events associated with tumorigenesis and the immune microenvironment. 2019, 9:7648-7665.

20. S B, S A, L G, P D, H S, J P, P B, V D, T S, J C, et al: ImmPort: disseminating data to the public for the future of immunology.Immunologic research 2014, 58:234-239. 
21. Y Z, B Z, L P, M C, AH K, O T, C B, SK C: Metascape provides a biologist-oriented resource for the analysis of systemslevel datasets. Nature communications 2019, 10:1523.

22. XY S, SZ Y, HP Z, J L, WZ G, SJ Z: A signature of 33 immune-related gene pairs predicts clinical outcome in hepatocellular carcinoma.Cancer medicine 2020.

23. $Y X, L L, Q B, Y X, Y Q, J W, J$ G: Individualized immune-related gene signature predicts immune status and oncologic outcomes in clear cell renal cell carcinoma patients.Urologic oncology 2020, 38:7.e1-7.e8.

24. S E, J B: Statistical predictions with glmnet.Clinical epigenetics 2019, 11:123.

25. PJ H, T L, MS P: Time-dependent ROC curves for censored survival data and a diagnostic marker.Biometrics 2000, 56:337-344.

26. AM N, CL L, MR G, AJ G, W F, Y X, CD H, M D, AA A: Robust enumeration of cell subsets from tissue expression profiles. Nature methods 2015, 12:453-457.

27. OC K, M W, S D: Metastatic lymph node ratio successfully predicts prognosis in western gastric cancer patients.Surgical oncology 2015, 24:84-88.

28. $L Z, P Z, Y T, Y$ W, H M, X X, Y Z, X Z, F G, P S: An immune-related gene pairs signature predicts overall survival in serous ovarian carcinoma.OncoTargets and therapy 2019, 12:7005-7014.

29. W D, G S, J Q, X Z, Q G: Aberrant expression of B7-H3 in gastric adenocarcinoma promotes cancer cell metastasis. Oncology reports 2014, 32:2086-2092.

30. Arigami T, Uenosono Y, Hirata M, Hagihara T, Yanagita S, Ishigami S, Natsugoe S: Expression of B7-H4 in blood of patients with gastric cancer predicts tumor progression and prognosis. Journal of Surgical Oncology 2010, 102:748752.

31. C Y, Y Z: Development and validation of prognostic nomogram for young patients with gastric cancer.Annals of translational medicine 2019, 7:641.

32. J L, ZF Z, W W, JW X, JB W, JX L, QY C, LL C, M L, RH T, et al: A novel TNM staging system for gastric cancer based on the metro-ticket paradigm: a comparative study with the AJCC-TNM staging system.Gastric cancer : official journal of the International Gastric Cancer Association and the Japanese Gastric Cancer Association 2019, 22:759768.

33. Z W, Z Y, B Z, Z R, Y Z, J L, L Y, Y Z, B Y, T W, J G: Identification of a 5-gene signature for clinical and prognostic prediction in gastric cancer patients upon microarray data.Medical oncology (Northwood, London, England) 2013, 30:678.

34. AC W, K T, H T, L Y, N D, M N, SJ L, JJ B, E O-T, P M, et al: Identification of a TLR2-regulated gene signature associated with tumor cell growth in gastric cancer.Oncogene 2017, 36:5134-5144.

35. H W, X W, Y C: Stromal-Immune Score-Based Gene Signature: A Prognosis Stratification Tool in Gastric Cancer.Frontiers in oncology 2019, 9:1212.

36. B J, Q S, Y T, Y W, H M, XX, Y Z, X Z, F G, P S: An immune-related gene signature predicts prognosis of gastric cancer.Medicine 2019, 98:e16273.

37. JA E, J S, D G, ND P: Relative expression analysis for molecular cancer diagnosis and prognosis. Technology in cancer research \& treatment 2010, 9:149-159.

38. Popovici V, Budinska E, Tejpar S, Weinrich S, Estrella H, Hodgson G, Van Cutsem E, Xie T, Bosman FT, Roth AD: Identification of a poor-prognosis BRAF-mutant-like population of patients with colon cancer.J Clin Oncol 2012, 30:1288-1295.

39. M B-K, K W, J B, A S, M S, M S: Elevated level of some chemokines in plasma of gastric cancer patients. CentralEuropean journal of immunology 2016, 41:358-362. 
40. X C, R C, R J, Z H: The role of CXCL chemokine family in the development and progression of gastric cancer.International journal of clinical and experimental pathology 2020, 13:484-492.

41. Z S, S C, C L, Y C, J J, WG J, L Y: Increased Expression of Gremlin1 Promotes Proliferation and Epithelial Mesenchymal Transition in Gastric Cancer Cells and Correlates With Poor Prognosis of Patients With Gastric Cancer.Cancer genomics \& proteomics 2020, 17:49-60.

42. Z X, C Z, A C, J L, H Z, W Z, X Z: Immune-related key gene CLDN10 correlates with lymph node metastasis but predicts favorable prognosis in papillary thyroid carcinoma.Aging 2020, 12:2825-2839.

43. $X \mathrm{~L}, \mathrm{~S} W, Y \mathrm{Y}, \mathrm{M} \mathrm{Z}, \mathrm{G} \mathrm{Z}, \mathrm{Z} \mathrm{H}$ : The prognostic landscape of tumor-infiltrating immune cell and immunomodulators in lung cancer.Biomedicine \& pharmacotherapy = Biomedecine \& pharmacotherapie 2017, 95:55-61.

44. P Y, YX F: Tumor-infiltrating T lymphocytes: friends or foes? Laboratory investigation; a journal of technical methods and pathology 2006, 86:231-245.

45. Y C, JW K, KH N, SH H, JW K, SH A, DJ P, KW L, HS L, HH K: Systemic inflammation is associated with the density of immune cells in the tumor microenvironment of gastric cancer.Gastric cancer : official journal of the International Gastric Cancer Association and the Japanese Gastric Cancer Association 2017, 20:602-611.

46. J D, J L, S L, X F, S C, Z Z, Y C, X Z: Prognostic potential of an immune score based on the density of CD8 T cells, CD20 B cells, and CD33/p-STAT1 double-positive cells and HMGB1 expression within cancer nests in stage IIIA gastric cancer patients. Chinese journal of cancer research = Chung-kuo yen cheng yen chiu 2016, 28:543-552.

47. H S, K M, Y K, Y M, H K, T M, Y F, S T, T O, Y F: Decreased Serum Concentration of Total IgG Is Related to Tumor Progression in Gastric Cancer Patients. Yonago acta medica 2017, 60:119-125.

48. Jackute J, Zemaitis M, Pranys D, Sitkauskiene B, Miliauskas S, Vaitkiene S, Sakalauskas R: Distribution of M1 and M2 macrophages in tumor islets and stroma in relation to prognosis of non-small cell lung cancer.BMC immunology 2018, 19:3.

49. Zhang B, Yao G, Zhang Y, Gao J, Yang B, Rao Z, Gao J: M2-polarized tumor-associated macrophages are associated with poor prognoses resulting from accelerated lymphangiogenesis in lung adenocarcinoma.Clinics 2011, 66:18791886.

50. C D, R G, C W, A W, M Q, J H, Y S, AW L, J L: Concordance of immune checkpoints within tumor immune contexture and their prognostic significance in gastric cancer.Molecular oncology 2016, 10:1551-1558.

51. R S, A Q, ALK T, S Z, CCY N, BT T, J L, KM K, P T: Transcriptional analysis of immune genes in Epstein-Barr virusassociated gastric cancer and association with clinical outcomes.Gastric cancer : official journal of the International Gastric Cancer Association and the Japanese Gastric Cancer Association 2018, 21:1064-1070.

52. Giraldo NA, Becht E, Pages F, Skliris G, Verkarre V, Vano Y, Mejean A, Saint-Aubert N, Lacroix L, Natario I: Orchestration and prognostic significance of immune checkpoints in the microenvironment of primary and metastatic renal cell cancer.Clinical Cancer Research 2015, 21:3031-3040.

53. HB J, PJ S, SC L, RM S, A A, S F, TL W, RL F: CTLA-4冈 Regulatory T Cells Increased in Cetuximab-Treated Head and Neck Cancer Patients Suppress NK Cell Cytotoxicity and Correlate with Poor Prognosis.Cancer research 2015, 75:2200-2210.

54. AR N, C S, R B, MW K, MF B, JR S: Predicting individual survival after gastric cancer resection: validation of a U.S.derived nomogram at a single high-volume center in Europe.Annals of surgery 2006, 243:74-81.

55. Y M, K A, T I, H I, A N, H Y, N T, SI K, T W: Nomogram for 5-year relapse-free survival of a patient with advanced gastric cancer after surgery. International journal of surgery (London, England) 2016, 35:153-159.

56. Z L, H C: Construction of a nomogram for the prediction of prognosis in patients with resectable gastric cancer undergoing fewer than sixteen lymph node biopsies. OncoTargets and therapy 2019, 12:7415-7428. 
57. C W, N W, H Z, T W, D Z: Development and validation of a nomogram to individually predict survival of young patients with nonmetastatic gastric cancer: A retrospective cohort study.Saudi journal of gastroenterology : official journal of the Saudi Gastroenterology Association 2019, 25:236-244.

58. Y H, M M, W S, Z H, L Z, X W: Development and validation of a prognostic nomogram in gastric cancer with hepatitis $B$ virus infection.Journal of translational medicine 2019, 17:98.

59. $M R, A B, L S, M G, C E O, M R, I B, C P, R F, D L$, et al: Prognosis of elderly gastric cancer patients after surgery: a nomogram to predict survival.Medical oncology (Northwood, London, England) 2018, 35:111.

60. B B, C Z, B Y, Y J, K H, Z L, X Z, S Y, X Z, Y F, et al: Identification of Subtype-Specific Three-Gene Signature for Prognostic Prediction in Diffuse Type Gastric Cancer.Frontiers in oncology 2019, 9:1243.

61. K P, E C, W L, X C, Y Y, Y C, Q L, Y W, X X, C T, et al: A 16-mRNA signature optimizes recurrence-free survival prediction of Stages II and III gastric cancer.Journal of cellular physiology 2020, 235:5777-5786.

62. SL W, MB F, EB K, SS A, TJ A, C A, CM B, BS B, A C, KA D, et al: Sentinel Lymph Node Biopsy and Management of Regional Lymph Nodes in Melanoma: American Society of Clinical Oncology and Society of Surgical Oncology Clinical Practice Guideline Update.Journal of clinical oncology : official journal of the American Society of Clinical Oncology 2018, 36:399-413.

\section{Figures}
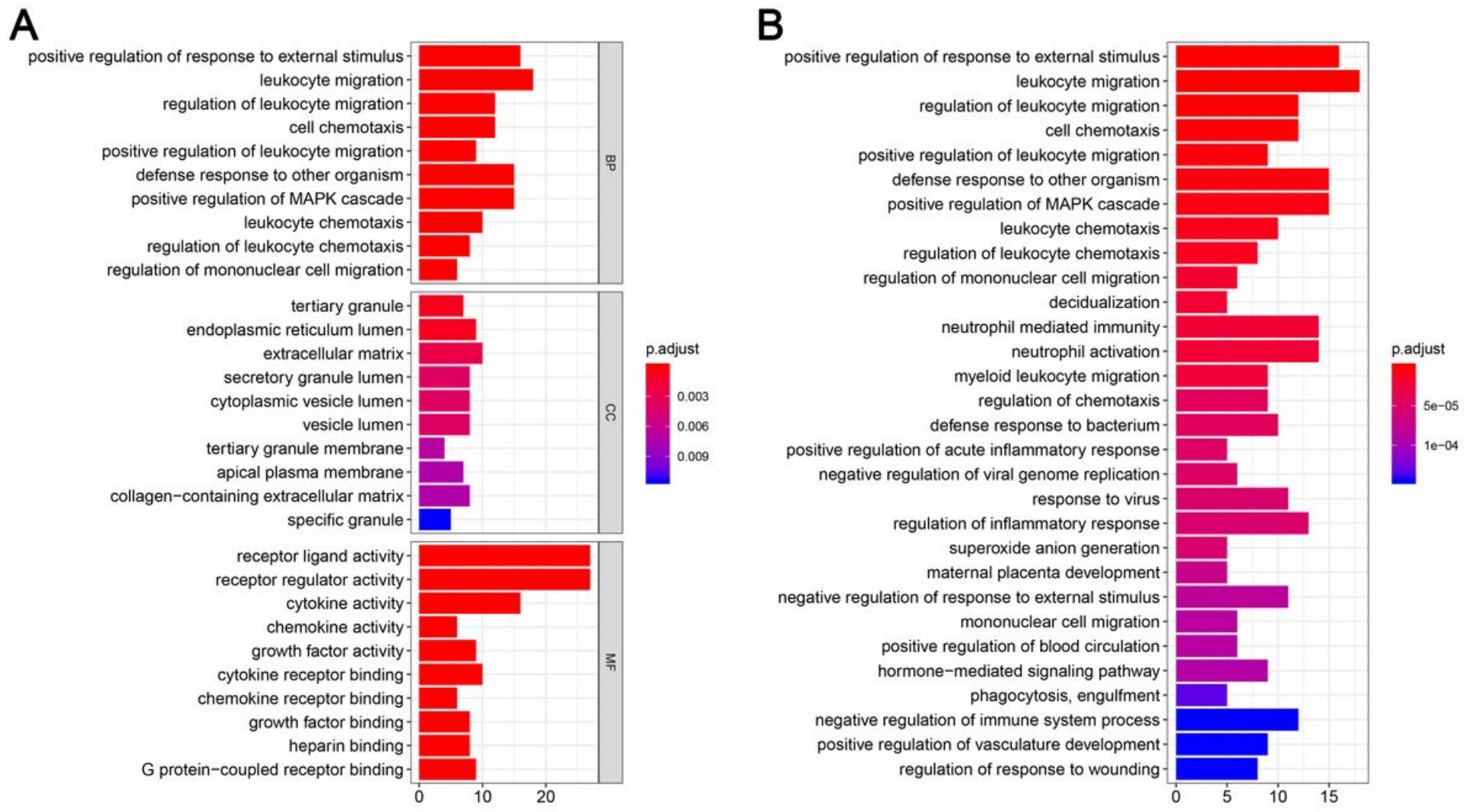

Figure 1

Establishment of the prognostic model for OS based on IRGPS. (A) Kaplan-Meier of the patients in the OS-prognostic signature; (B) time-dependent ROC curve analysis of the OS-prognostic signature; (C) risk score distribution and survival status scatter plots of GC patients. 
A
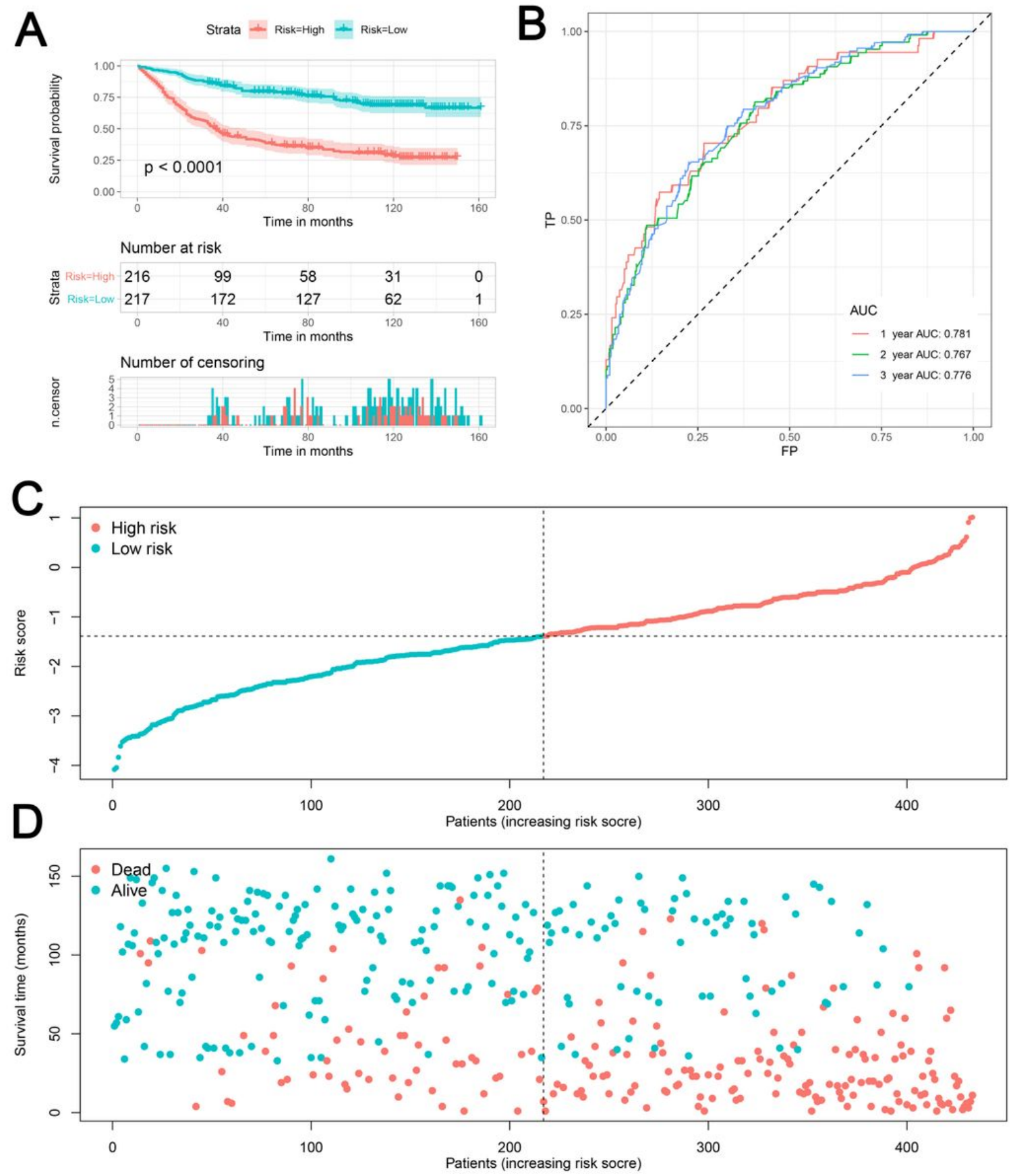

Figure 2

Kaplan-Meier curves of OS and RFS among independent validation cohorts. (A, D) GSE13861; (B, E) GSE26901; (C, F) GSE66229. 

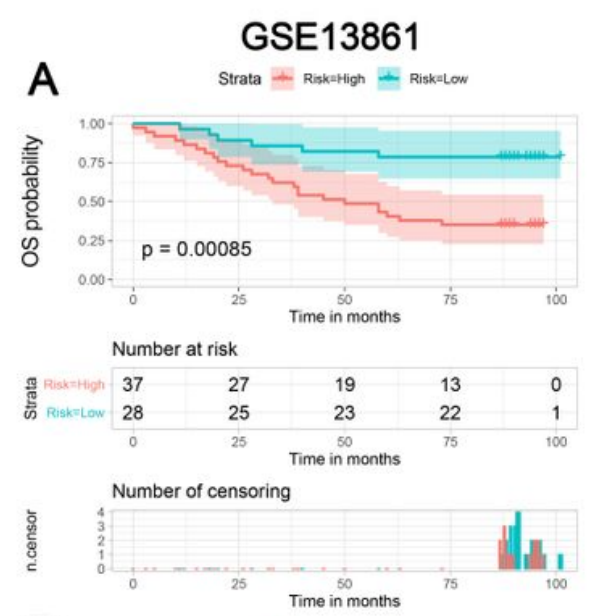

\section{D}

$$
\text { Strata } 4 \text { Rakk=High } \perp \text { Risk=Low }
$$
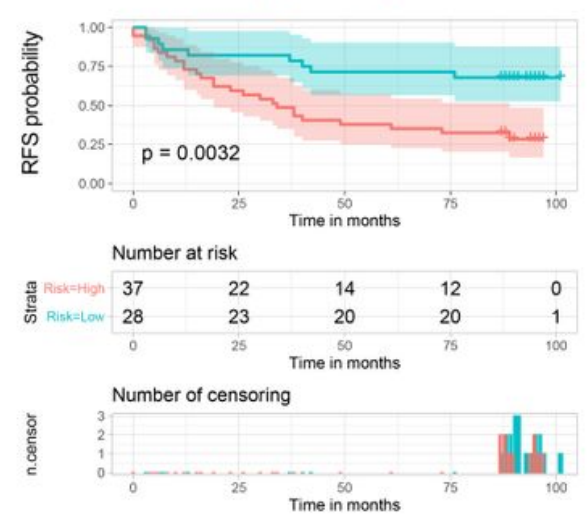

GSE26901
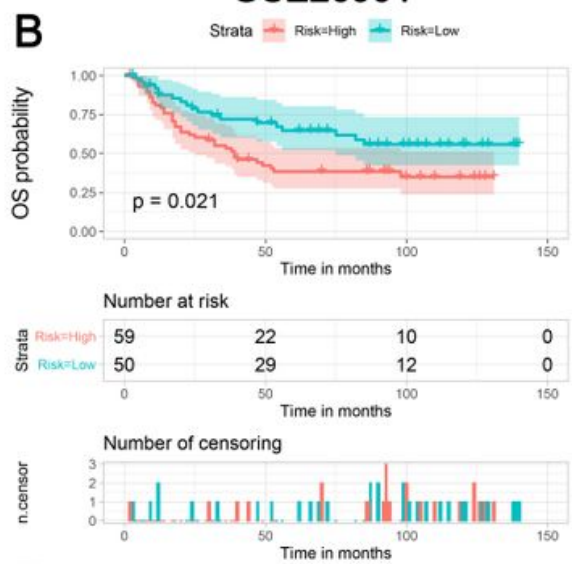

E
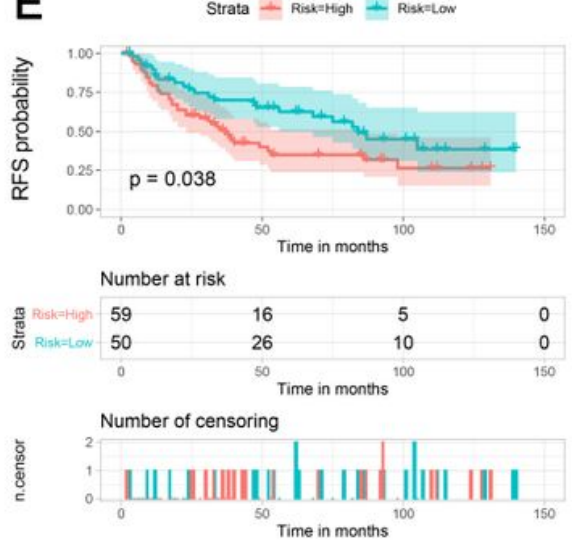
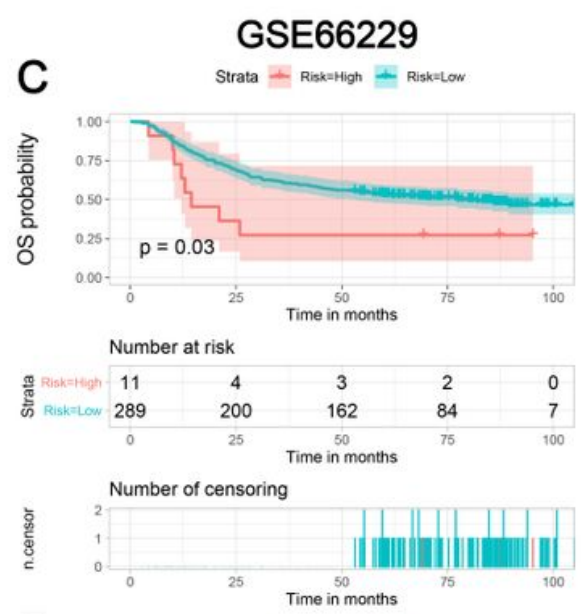

F $\quad$ Strata - Risk=High $\div$ Risk=Low
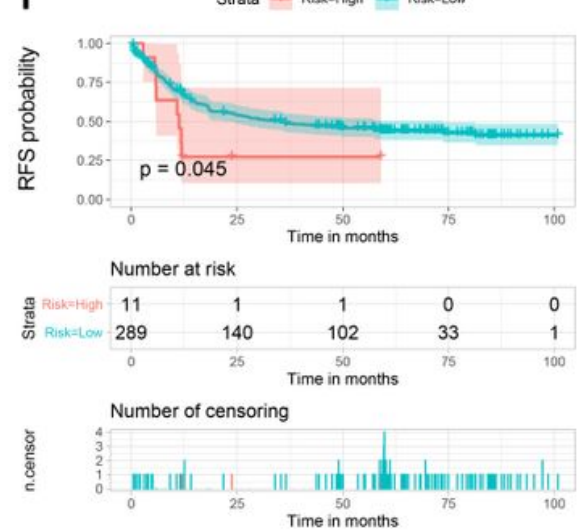

\section{Figure 3}

The distribution of IRGPs that significantly associated with immune cells and immune checkpoints. (A) the differences among immune cells in high- and low-groups; (B) the differences among immune checkpoints in high- and low-groups; (C) the differences among IRGPs in high- and low-groups; (D) the annotated Circos diagram of IRGs. The outer circle indicates the position of IRGs in the chromosome. The inner circle represents the potential interaction between IRGs. 
A

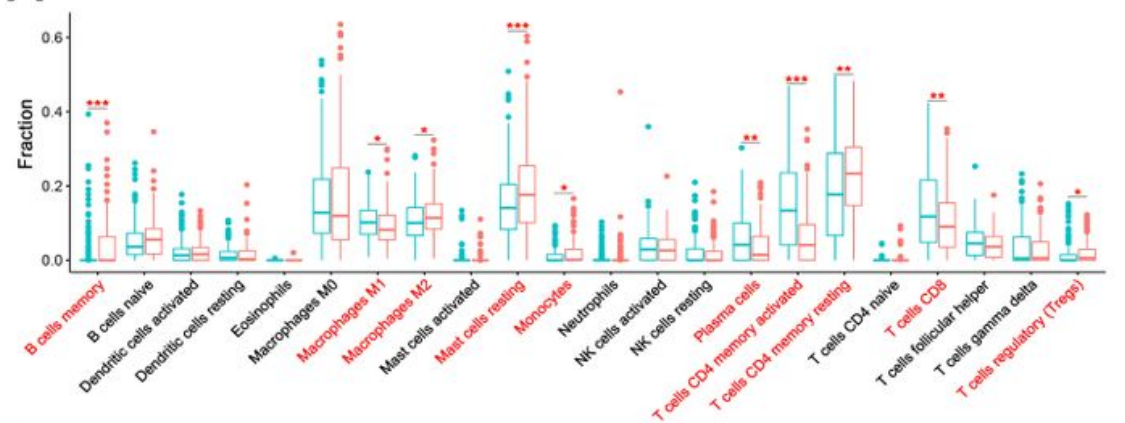

C

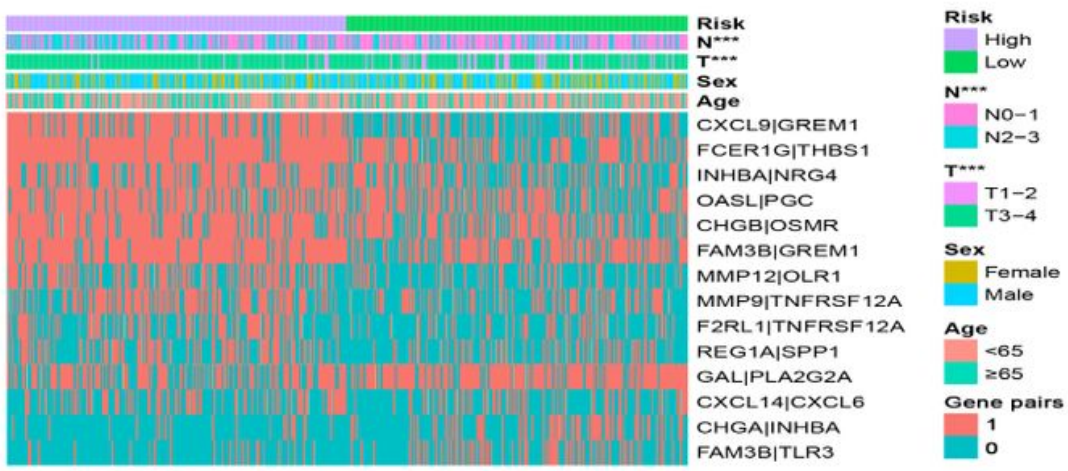

B

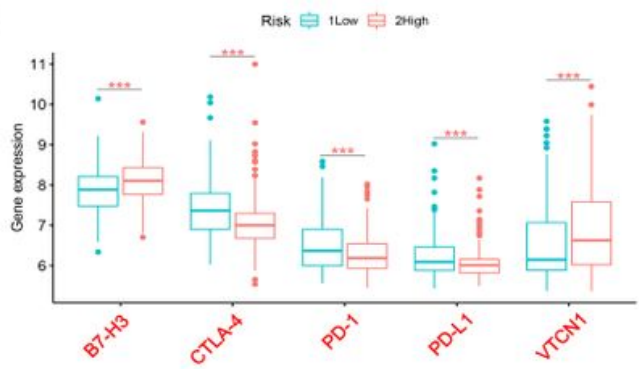

D

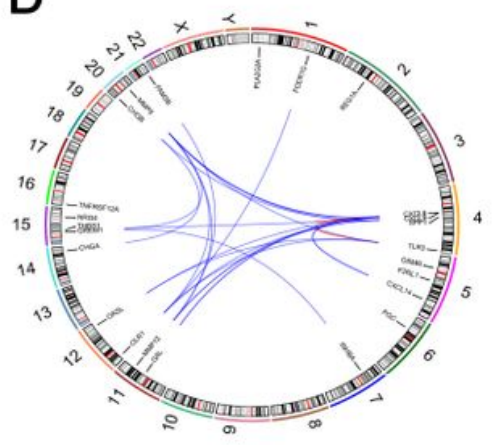

\section{Figure 4}

Development of an IRGPs-clinical nomogram. (A) univariate and multivariate Cox regression analysis of clinical factors.

(B) a nomogram for the prediction of the OS of GC patients at 1-, 2-, and 3-year; (C) calibration curves of the nomogram; (D-F) DCA of the nomogram for 1-, 2-, and 3-years in the GC cohort. 
A

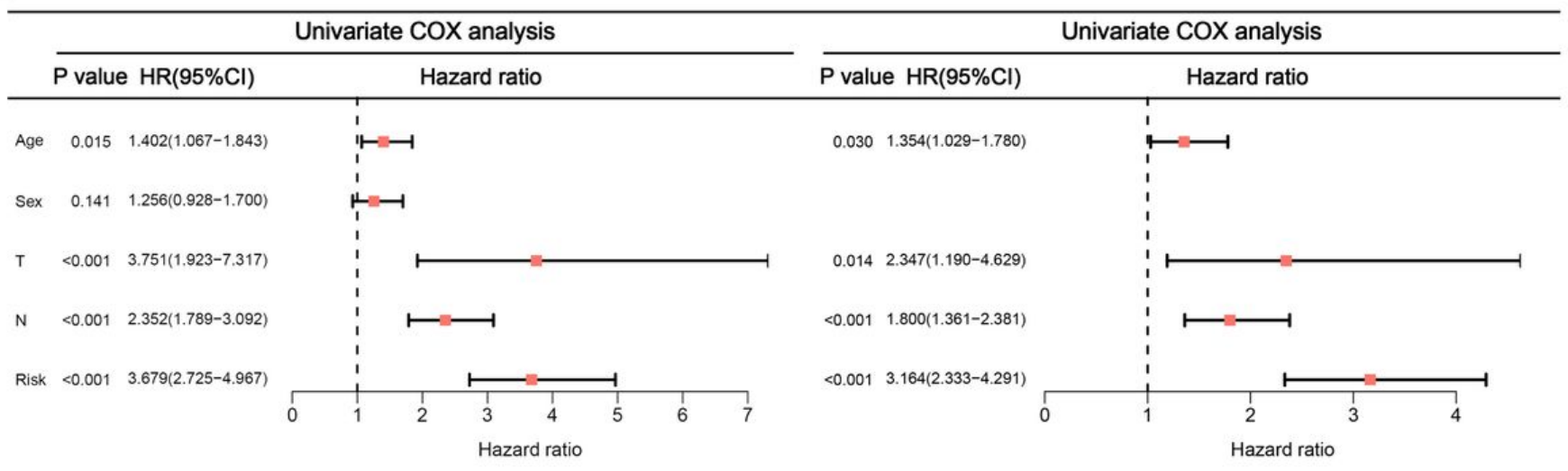

B

Points

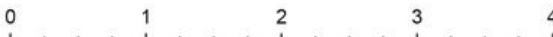

4

$6 \quad 7$

Age

T

N

$<65$

$1-2$

Risk

Total Points

1 year OS Probability

2 year OS Probability

3 year OS Probability
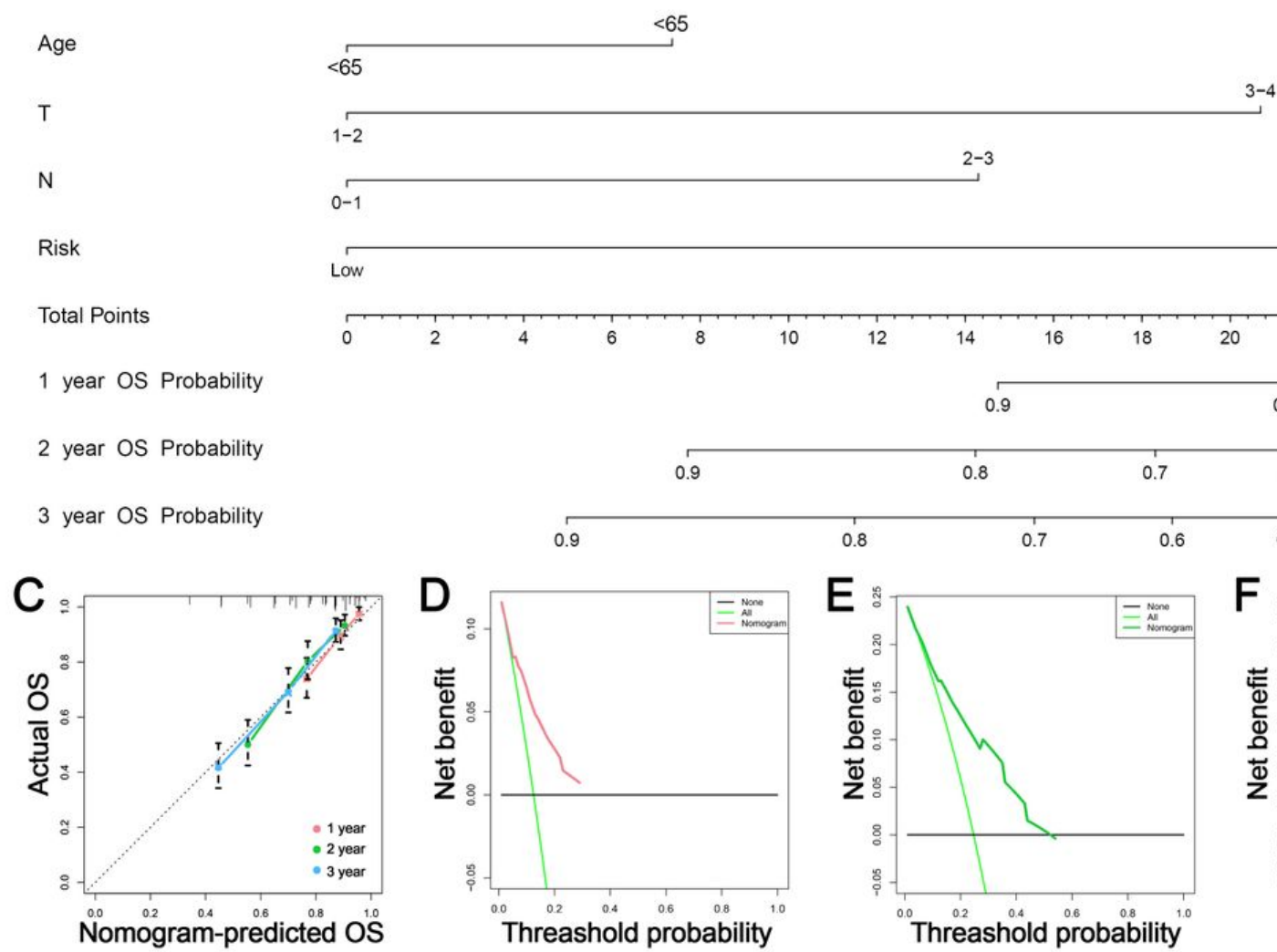

$0-1$
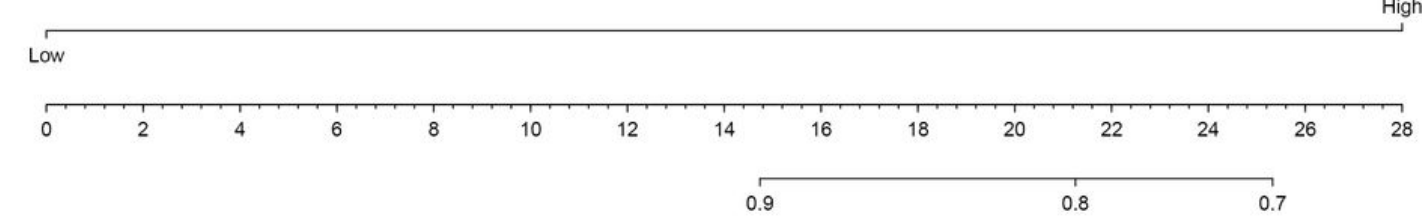

A 
- Supplementaryfile2.xIsx

- Supplementaryfile1.xlsx

- SupplementaryFig4.tif

- SupplementaryFig3.tif

- SupplementaryFig2.tif

- SupplementaryFig1.tif

- Supplementaryfile7.xlsx

- Supplementaryfile6.xlsx 